\title{
Clinical Characteristics of Ischemic Colitis According to Location
}

\author{
Ho Jin Chang, Chul Woon Chung, Kwang Hyun $\mathrm{Ko}^{1}$, Jong Woo Kim \\ Departments of Surgery and ${ }^{1}$ Internal Medicine, CHA Bundang Medical Center, CHA University School of Medicine, Seongnam, Korea
}

Purpose: The aim of this study was to analyze various clinical characteristics of ischemic colitis according to its location. Methods: The medical records of 92 cases of gastrointestinal ischemic colitis (IC) diagnosed at Bundang CHA Hospital from 1995 to 2008 were reviewed and analyzed retrospectively. The patients were diagnosed by using colonoscopic biopsies or laparotomy findings. The patients were divided into two groups, right and left, according to the main involvement area of the IC at the embryologic boundary line of the distal transverse colon, and the two groups were compared as to clinical characteristics and co-morbid diseases.

Results: Left IC was present in 59 patients (64.1\%) and right IC in 33 patients (35.9\%). No differences between the two groups in terms of clinical characteristics, cardiovascular disease and diabetes mellitus were observed. However, in 16 cases with renal failure, 10 patient had right IC and 6 patients had left IC, and this difference had statistical significance $(\mathrm{P}=$ $0.014)$. Among the 16 , the 11 patients requiring hemodialysis included 8 with right IC $(24.2 \%)$ and 3 with left IC $(5.1 \% ; \mathrm{P}=$ 0.009). Among the 19 cases of severe IC requiring surgical treatment or involving mortality, irrespective of surgery, 11 patients showed right IC and 8 patients showed left IC $(\mathrm{P}=0.024)$.

Conclusion: Right-side ischemic colitis was significantly associated with renal failure and disease severity, so patients with right-side colon ischemia should be more carefully observed and managed.

Keywords: : Colitis, Ischemic; Localization; Severity

\section{INTRODUCTION}

Ischemic colitis emanates from insufficient blood supply to the colon, and it causes subsequent inflammation and damage, which progressively may result in colonic necrosis. It is the most common type of bowel ischemia, approaching an approximately $50 \%$ prevalence in the gastrointestinal tract. It frequently occurs in elderly women, mostly ages over 60 , although it can occasionally be found in young or middle-aged groups. The underlying causes of ischemic colitis include thrombosis or embolism in the mesenteric artery, vessel occlusion due to mechanical causes such as bowel obstruc-

Received: June 9, 2011 Accepted: October 5, 2011

Correspondence to: Jong Woo Kim, M.D.

Department of Surgery, CHA Bundang Medical Center, 351 Yatap-dong,

Bundang-gu, Seongnam 463-712, Korea

Tel: +82-31-780-5250, Fax: +82-31-780-5259

E-mail: kjw@cha.ac.kr

(c) 2011 The Korean Society of Coloproctology

This is an open-access article distributed under the terms of the Creative Commons Attribution NonCommercial License (http://creativecommons.org/licenses/by-nc/3.0) which permits unrestricted noncommercial use, distribution, and reproduction in any medium, provided the original work is properly cited. tion, transient hypotension during or after surgery, hypoperfusion due to congestive heart failure, septic shock etc. Hypertension, diabetes mellitus, ischemic heart disease, congestive heart disease, and hyperlipidemia are known to be risk and contributing factors. The incidence of ischemic colitis may be underestimated due to the broad spectrum of diverse clinical courses. Mild clinical presentation may lead to misdiagnosis as inflammatory bowel disease or infectious colitis [1]. In mild clinical presentation, only the mucosal or the submucosal layer is affected, causing mild abdominal pain or diarrhea. Such symptoms are relieved before arrival at a medical facility, and conservative care can improve the condition. However, severe clinical presentation exhibits transmural involvement of the colon, causing fulminant necrotizing colitis. Such cases mostly require emergent surgical correction. Delayed surgical treatment or inadequate intervention can evoke sepsis and septic shock, life-threatening situations [2].

Ischemic colitis may occur in any part of the the colon. It has been reported that the involved site of colon might determine the disease's severity, symptomatic differences, and prognosis. According to Sotiriadis et al. [2], despite being only $10 \%$ of all ischemic colitis cases, patients with ischemic colitis confined to the right colon 
mainly showed severe clinical presentation, and more than 50\% of them needed surgery. In general, hematochezia has been considered to be the most common complaint in ischemic colitis. However, patients with ischemic colitis localized on the right side of colon present with abdominal pain as their chief complaint. Furthermore, those patients showed more severe clinical manifestation, and more than $50 \%$ of the total number of patients required emergent surgical correction. Among those who received surgical treatment, more than $20 \%$ expired. In this study, we investigated the clinical characteristics of ischemic colitis diagnosed at Bundang $\mathrm{CHA}$ hospital. We also looked into how the known risk factors, such as diabetes, cardiovascular diseases, or chronic renal failure, were related to the occurrence of either right-sided or left-sided ischemic colitis.

\section{METHODS}

Retrospectively, we reviewed the cases of 92 patients diagnosed as having ischemic colitis from 1995 to 2008. Diagnoses were made by means of colonoscopy, biopsy, and pathologic examination of specimens obtained intraoperatively. The 92 patients included 10 that were diagnosed using pathologically confirmed tissue samples obtained during surgery and 82 that were suspected of having ischemic colitis based on X-ray or abdominal computed tomography (CT) scan or angiography and finally diagnosed by using colonoscopic appearance and biopsy result. Endoscopic findings were petechiae, mucosal exudates, hemorrhage, or ulceration, which were confirmed by board-certified gastroenterologists. The analyses were made based on the comments and opinions written by the gastroenterologists. Also, pathologic findings were analyzed based on diagnoses and comments by board-certified pathologists. For some cases, two or more diagnostic procedures were conducted.

In this study, only ischemic colitis caused by hypoperfusion or thromboembolism was considered. Ischemic colitis caused by intestinal obstruction, such as volvulus or colon cancer, was excluded. The sample patients were divided into two groups, right and left, according to the main involvement area of the ischemic colitis at the embryologic boundary line of the distal transverse colon. The two groups were compared and analyzed by age, sex, and co-morbid diseases. Looking into cardiovascular diseases, of the groups were compared to find correlations with accompanying hypertension, atrial fibrillation, heart failure, or ischemic heart diseases. Furthermore, accompanying diabetes mellitus, cardiovascular diseases, and renal failure were analyzed to determine whether each disease was related to ischemic colitis. Severe ischemic colitis was defined as cases requiring surgical treatment or expired cases, and those were analyzed separately. Surgical indications were peritoneal irritation signs such as rigidity and tenderness with systemic inflammatory response syndrome with more than 2 items among systemic blood pressure $<90 \mathrm{mmHg}$, pulse rate $>90 /$ minute, respiratory rate $>20 /$ minute, and white blood cell count $>12,000$ / $\mathrm{mm}^{3}$ or $<4,000 / \mathrm{mm}^{3}$. Most mild cases were treated with conser- vative manage.

The statistical analysis applied Window SPSS ver. 12.0 (SPSS Inc., Chicago, IL, USA). In order to analyze the differences and similarities in right-sided and left-sided ischemic colitis, the chi-square test and Fisher's test were used. Statistical significance was only considered when the P-value was less than 0.05 .

\section{RESULT}

\section{Characteristics of sample patients}

This study was comprised of 29 males and 64 females, a total of 92 patients. The mean age was 64.2 for males and 68.1 for females, 66.9 overall. The number of cases presenting only left-sided ischemic colitis was 59 (64.1\%) whereas the number for right-sided ischemic colitis was 33 (35.9\%). Seven cases in which the entire colon was affected by ischemia were excluded in the analysis because of their being inconsistent with the purpose of this article. The overall clinical characteristics are shown in the Table 1.

Analyzing the sample patients according to the involved segment in the colon, the mean age of the patients with left-sided colitis was 66.3 whereas the mean age of the patients with right-sided colitis was 68.4. Dividing into two groups by age - over 65 and under 65, we didn't find any statistically significant correlation between age and the affected region. Also, an analysis on the correlation between sex and the affected segment provided no statistically significant result (Table 2).

\section{Correlation with cardiovascular disease}

Considering cardiovascular diseases, well-known risk factors of ischemic colitis, we investigated its correlation with the affected segment in the colon. Cardiovascular diseases were divided into hypertensive disease, atrial fibrillation, arrhythmia, heart failure, and ischemic heart disease. A total of 56 patients were diagnosed

Table 1. Clinical characteristics of patients with ischemic colitis

\begin{tabular}{lc}
\hline Characteristics & Total $(\mathrm{n}=92)$ \\
\hline Mean age $(\mathrm{yr})$ & 66.9 \\
Male & $29(31.50)$ \\
Female & $63(68.50)$ \\
Coexisting conditions & \\
Hypertension & $48(52.2)$ \\
Atrial fibrillation & $7(7.6)$ \\
Congestive heart failure & $5(5.4)$ \\
Ischemic heart disease & $7(7.6)$ \\
Diabetes Mellitus & $20(21.7)$ \\
Renal Failure & $16(17.4)$ \\
$\quad$ On dialysis & $11(12.0)$ \\
$\quad$ Without dialysis & $5(5.4)$ \\
\hline
\end{tabular}

Values are presented as number (\%) unless otherwise indicated. 
with cardiovascular diseases: 48 with hypertension, 7 with ischemic heart disease ( 5 with angina and 2 with an acute myocardial infarction), 7 with atrial fibrillation, and 5 with congestive heart failure. Some patients had two or more such diseases. Evaluating correlations between the affected area of the colon in ischemic colitis and the cardiovascular diseases listed above, we observed 22 cases of right-sided ischemic colitis $(66.7 \%)$ and 34 cases of leftsided ischemic colitis (57.6\%), but this result demonstrated no statistical significance. Furthermore, comparing patients with hypertension or without hypertension, the number of patients with rightsided colitis was $20(60.6 \%)$ whereas the number with left-sided colitis was 28 (47.5\%), and this result had no statistical significance as well. Analyzing the correlation with atrial fibrillation, 5 out of the total 33 right-sided ischemic colitis (15.2\%) and 2 out of the total 59 left-sided ischemic colitis had atrial fibrillation. The P-value was 0.054 , which might be considered as borderline statistically significant. The analysis on the correlation with ischemic colitis demonstrated 1 case of right-sided ischemic colitis (3.0\%) and 6 of left-sided colitis (10.2\%), but no statistical significance was ob-

Table 2. Clinical characteristics of patients according to the location of ischemic colitis

\begin{tabular}{lcccc}
\hline Characteristics & $\begin{array}{c}\text { Right } \\
(\mathrm{n}=33)\end{array}$ & $\begin{array}{c}\text { Left } \\
(\mathrm{n}=59)\end{array}$ & $\begin{array}{c}\text { Total } \\
(\mathrm{n}=92)\end{array}$ & P-value \\
\hline Mean age (yr) & 68.45 & 66.30 & 66.93 & 0.253 \\
$\quad$ Above 65 & $23(20.45)$ & $34(36.55)$ & $57(57)$ & \\
$\quad$ Below 65 & $10(12.55)$ & $25(22.45)$ & $35(35)$ & \\
Sex & & & & 0.261 \\
$\quad$ Male & $8(24.2)$ & $21(35.6)$ & $29(31.5)$ & \\
$\quad$ Female & $25(75.8)$ & $38(64.4)$ & $63(68.5)$ & \\
\hline
\end{tabular}

Values are presented as number (\%) unless otherwise indicated.

Table 3. Relationship of cardiovascular disease, diabetes mellitus, renal failure and disease severity according to the location of ischemic colitis

\begin{tabular}{lccc}
\hline Variables & Right $(\mathrm{n}=33)$ & Left $(\mathrm{n}=59)$ & P-value \\
\hline Cardiovascular disease & $22(66.7)$ & $34(57.6)$ & 0.394 \\
Hypertension & $20(60.6)$ & $28(47.5)$ & 0.225 \\
Atrial fibrillation & $5(15.2)$ & $2(3.4)$ & 0.054 \\
Congestive heart failure & $3(9.1)$ & $2(3.4)$ & 0.244 \\
Ischemic heart disease & $1(3.0)$ & $6(10.2)$ & 0.209 \\
Diabetes mellitus & $7(21.2)$ & $13(22.0)$ & 0.927 \\
Renal failure $(\mathrm{a}+\mathrm{b}+\mathrm{c})$ & $10(30.3)$ & $6(10.2)$ & 0.014 \\
CRF + ESRD & $8(24.2)$ & $5(8.5)$ & 0.040 \\
Hemodialysis & $8(24.2)$ & $3(5.1)$ & 0.009 \\
Severe disease & $11(33.3)$ & $8(13.6)$ & 0.024 \\
\hline
\end{tabular}

Values are presented as number (\%).

a, acute renal failure; b, chronic renal failure (CRF); c, end-stage renal disease (ESRD). served. Reviewing the correlation with the heart failure, the data indicated 3 patients (9.1\%) had right-sided ischemic colitis, and 2 patients (3.4\%) had left-sided ischemic colitis (Table 3).

\section{Correlation with diabetes mellitus}

We evaluated the correlation between the affected area in the colon and diabetes mellitus. Among the total of 92 sample patients, 20 patients $(21.7 \%)$ were diagnosed as having type I or II diabetes. Among those patients with diabetes, 7 patients showed right-sided ischemic colitis (21.2\%) while 13 patients exhibited left-sided colitis (22.0\%), but no statistical significance was noted (Table 4 ).

\section{Correlation with renal failure}

Out of the total 92 patients with ischemic colitis, 6 patients were diagnosed as having end-stage renal disease (ESRD), and 7 patients as having chronic renal failure (CRF). Sixteen patients (17.4\%) had been previously diagnosed as having acute renal failure (ARF), including 3 cases of acute renal failure preceding ischemic colitis. The fundamental diseases of ESRD and CRF were mostly diabetes mellitus, glomerulonephropathy and cardiovascular diseases, such as hypertension etc. However, two patients who developed acute renal failure subsequent to ischemic colitis were excluded from this study. Among the patients suffering from ischemic colitis along with preceding renal failure, 10 patients $(30.3 \%)$ showed right-sided ischemic colitis, and 6 patients (10.2\%) demonstrated left-sided ischemic colitis. This result was statistically significant, $\mathrm{P}=0.014$ (Table 5). Only considering ESRD and CRF patients, excluding the three ARF patients, we observed a statistically significant result for a greater incidence of right sided ischemic colitis (8 right-sided, $24.2 \%$ and 5 left-sided ischemic colitis, $8.5 \%, \mathrm{P}=0.04)$. The 11 patients requiring hemodialysis, from among the 16 patients, even showed an ischemic condition of the colon (8 right-sided ischemic colitis, $24.2 \%$ and 3 left-sided ischemic colitis, 5.1\%) with greater statistical significance $(P=0.009$, Table 6).

Table 4. The relationship of diabetes mellitus according to the location of ischemic colitis

\begin{tabular}{lrcc}
\hline Variables & \multicolumn{1}{c}{ Right } & Left & P-value \\
\hline Diabetes Mellitus (+) & $7(21.2)$ & $13(22.0)$ & 0.9269 \\
Diabetes Mellitus (-) & $26(78.8)$ & $46(78.0)$ & \\
\hline
\end{tabular}

Values are presented as number (\%).

Table 5. The relationship of renal failure according to the location of ischemic colitis

\begin{tabular}{lccc}
\hline & Right & Left & P-value \\
\hline Renal failure $(a+b+c)$ & $10(30.3)$ & $6(10.2)$ & 0.014 \\
CRF + ESRD & $8(24.2)$ & $5(8.5)$ & 0.040 \\
\hline
\end{tabular}

Values are presented as number (\%).

$\mathrm{ARF}$, acute renal failure; CRF, chronic renal failure; ESRD, end stage renal disease. 
Table 6. The relationship of hemodialysis according to the location of ischemic colitis

\begin{tabular}{lccc}
\hline & Right & Left & P-value \\
\hline On dialysis & $8(24.2)$ & $3(5.1)$ & 0.009 \\
Without dialysis & $2(6.1)$ & $3(5.1)$ & 0.793 \\
\hline
\end{tabular}

Values are presented as number (\%).

\section{Correlation with severity of the disease}

As mentioned earlier, severe ischemic colitis was defined as that requiring surgical treatment or resulting in death regardless modality of treatment. A total of 19 cases were recorded based on that definition. Among them, 10 patients received surgical treatment (1 segmental resection of the colon, 2 ileocecectomies, 4 right hemicolectomies, 1 anterior resection and 2 Hartmann's procedures), of which 3 patients died postoperatively. Nine patients expired without any surgical treatment.

The causes of death were mostly septic shock, multi-organ failure, acute respiratory distress syndrome and massive bleeding with hypovolemic shock. When the patients were divided into two groups, one group demonstrating ischemic colitis confined to the right and the other to the left side, 11 patients $(33.3 \%)$ showed right-sided colitis, and 8 patients (13.6\%) exhibited left-sided colitis. This was a statistically significant difference $(\mathrm{P}=0.024)$ (Table 7$)$.

\section{DISCUSSION}

Various underlying conditions are known to be risk factors for ischemic colitis, for example, hypertension, congestive heart failure, smoking, hypercholesterolemia, hyperlipidemia, past history of abdominal surgery, hypotension, shock, diabetes, and past history of cerebrovascular or cardiovascular accident. However, major vascular occlusion has not been observed in most cases, and often the exact cause has not been identified $[3,4]$. Ischemic colitis is frequently observed in the elderly. If it occurs at a younger age, coagulopathy, vasculitis, or use of oral contraceptive should be considered [5]. It demonstrates diverse clinical manifestations from spontaneous recovery or rapid clinical improvements to critical condition requiring emergent surgical treatment and even causing death. Therefore, determining poor prognostic factors may bolster the decision on treatment options [6].

Renal failure is well known to be an important risk factor, and it is also reported to be a risk factor for severe disease. Recently, its correlation with right-sided ischemic colitis has been emphasized $[1,2,7]$. Along with other previous studies, our study, with statistical significance, indicates a higher prevalence of right-sided ischemic colitis in patients with renal failure, especially those on hemodialysis. Ischemic colitis confined to the right colon should be related to renal failure in the aspect of blood flow because a hypovolemic condition might cause a prompt ischemic condition in the colon. Especially, long and straight blood vessels profoundly influence the blood supply. Thus, the right side of the colon seems to be
Table 7. The relationship of the disease severity according to the location of ischemic colitis

\begin{tabular}{lccc}
\hline & Right & Left & P-value \\
\hline Severe & $11(33.3)$ & $8(13.6)$ & 0.024 \\
Mild & $22(66.7)$ & $51(86.4)$ & \\
\hline
\end{tabular}

Values are presented as number (\%).

affected more easily [8]. Hemodialysis may predispose a patient to ischemia due to a greater perturbation in the blood flow [2]. This hypothesis supports the underlying diseases, such as hypertension or diabetes mellitus, which influence the blood perfusion pressure, having an effect on the ischemic region in the colon. Thus, supported by several reports, diseases affecting blood perfusion pressure, such as coronary disease, hypotension, or hypovolemia, possibly increase the prevalence of ischemic colitis confined to the right side $[7,9,10]$. Evidenced by statistical significance, our study indicates a higher mortality rate in patients who suffer from ischemic colitis with renal failure.

Patients with hypertension and diabetes demonstrated no differencse in the ischemic segment of the colon. Patients with atrial fibrillation, who may easily develop a thromboembolism, did not exhibit a higher incidence of ischemic colitis confined to the right side. Patients with myocardial ischemia showed statistically significant difference in the occurrence of right-sided ischemic colitis. Particularly, patients simultaneously suffering from angina pectoris or acute myocardial ischemia and ischemic colitis demonstrated a rather higher incidence rate of ischemic colitis confined to the left colon.

Whether the segment of the colon invaded by ischemic colitis is related to the prognosis or not has been a subject of controversy. Flobert et al. [7] reported that the involvement of the right colon in ischemic colitis might be related to the severity of the disease. Also, Sotiriadis et al. [2] reported that ischemic colitis invading the right colon had a five times greater rate of requiring surgical treatment and a twice higher mortality rate compared to colonic involvement in other regions. Ahn et al. [1] also reported that, among 18 patients suffering from ischemic colitis confined to the right side, 9 patients required surgical treatment, supporting the finding of a higher rate of surgical treatment than for ischemic colitis involving other segments of the colon. Similar to previous studies, our study demonstrated that ischemic colitis confined to the right colon required surgery and exhibited a higher mortality rate than ischemic colitis confined to the left side, and this difference was statistically significant (Table 3 ). The higher severity of right-sided ischemic colitis might also be related to vascular structure with long-segment, straight vessels which may be more vulnerable to ischemia, making ischemic colitis rapidly progressive.

In cases of renal failure, dialysis, which can cause hemodynamic change, may have a greater effect more than the renal failure itself. However, a study done by Anon et al. [11] on prognostic factors related to ischemic colitis showed that the segment affected by isch- 
emic colitis did not influence the prognosis. One recent Korean study done by Ahn et al. [1] reported that the severity of ischemic colitis was not affected by the involved segment in the colon. Such reports contradict previous study results. Such opposing results might have arisen from differences in the patient populations between the studies. The study done Anon et al. [11] included only 4 patients and the study done by Ahn et al. [1] included 9 patients with ischemic colitis confined to the right side. On the other hand, the study by Sotiriadis et al. [2], which concluded that invasion of the right colon was a poor prognostic factor, included 71 patients; 18 patients were included in the study by Lim et al. [12], and 33 patients were included in this study. Thus, the sample size might have caused the differences in the study results because relatively large sample sizes would have affected the statistical analyses.

A statistical analysis comparing ischemic colitis confined to the right colon to left-sided ischemic colitis showed that right-sided ischemic colitis required more surgical treatment and showed a higher mortality rate than left-sided ischemic colitis. Thus, patients with right-sided colitis, which might be more severe than left-sided colitis, should be more carefully observed and managed.

\section{CONFLICT OF INTEREST}

No potential conflict of interest relevant to this article was reported.

\section{REFERENCES}

1. Ahn SE, Lee HL, Cho SC, Yoon JH, Lee OY, Yoon BC, et al. Is end stage renal disease a poor prognosis factor of ischemic colitis?
Korean J Gastroenterol 2009;53:235-8.

2. Sotiriadis J, Brandt LJ, Behin DS, Southern WN. Ischemic colitis has a worse prognosis when isolated to the right side of the colon. Am J Gastroenterol 2007;102:2247-52.

3. Sreenarasimhaiah J. Diagnosis and management of intestinal ischaemic disorders. BMJ 2003;326:1372-6.

4. Gandhi SK, Hanson MM, Vernava AM, Kaminski DL, Longo WE. Ischemic colitis. Dis Colon Rectum 1996;39:88-100.

5. Brandt LJ, Boley SJ. Colonic ischemia. Surg Clin North Am 1992; 72:203-29.

6. Brandt LJ. Bloody diarrhea in an elderly patient. Gastroenterology 2005;128:157-63.

7. Flobert C, Cellier C, Berger A, Ngo A, Cuillerier E, Landi B, et al. Right colonic involvement is associated with severe forms of ischemic colitis and occurs frequently in patients with chronic renal failure requiring hemodialysis. Am J Gastroenterol 2000;95:195-8.

8. Scharff JR, Longo WE, Vartanian SM, Jacobs DL, Bahadursingh AN, Kaminski DL. Ischemic colitis: spectrum of disease and outcome. Surgery 2003;134:624-9.

9. Guttormson NL, Bubrick MP. Mortality from ischemic colitis. Dis Colon Rectum 1989;32:469-72.

10. Sakai L, Keltner R, Kaminski D. Spontaneous and shock-associated ischemic colitis. Am J Surg 1980;140:755-60.

11. Anon R, Bosca MM, Sanchiz V, Tosca J, Almela P, Amoros C, et al. Factors predicting poor prognosis in ischemic colitis. World J Gastroenterol 2006;12:4875-8.

12. Lim YJ, Son HJ, Kang TW, Kim GC, Lee MS, Lee JH, et al. Clinical patterns and prognostic factors of ischemic colitis. Korean J Gastrointest Endosc 2001;22:76-82. 